\title{
Relação entre a atividade inflamatória e o estado nutricional de pacientes com câncer de pulmão
}

\section{Relation between inflammatory activity and nutritional status of patients with lung cancer}

Benedita Jales Souzaํ. Armênia Uchôa de Mesquita². Amanda Rocha Meireles ${ }^{3}$. Juliana Girão de Brito ${ }^{3}$. Thalita Evangelista Bandeira ${ }^{4}$. José Aurillo Rocha 5 .

1 Universidade Estadual do Ceará (UECE), Hospital Dr. Carlos Alberto Studart Gomes, Fortaleza, Ceará, Brasil. 2 Universidade Estadual do Ceará (UECE), Universidade de Fortaleza (UNIFOR), Fortaleza, Ceará, Brasil. 3 Universidade Cruzeiro do Sul (UNICSUL), Fortaleza, Ceará, Brasil. 4 Universidade Estadual do Ceará (UECE), Fortaleza, Ceará, Brasil. 5 Universidade Federal do Ceará (UFC), Fortaleza, Ceará, Brasil

\section{RESUMO}

Objetivo: verificar a correlação entre a atividade inflamatória e o estado nutricional de pacientes com câncer de pulmão. Método: estudo transversal e quantitativo, realizado de março a outubro de $2015 \mathrm{com}$ pacientes com câncer de pulmão. Os pacientes foram avaliados pelo índice de massa corporal, circunferência do braço, dobra cutânea tricipital, circunferência muscular do braço, contagem total de linfócitos e pela relação proteína C-reativa/albumina. Foi utilizado o coeficiente de Spearman, considerando o nível de significância de 5\%. Resultados: participaram 58 indivíduos com idade média de 65,02 \pm 9,88 anos. A desnutrição foi maior pela dobra cutânea tricipital $(55,1 \%)$ e menor pelo índice de massa corporal $(37,9 \%)$. Correspondentemente, a depleção imunológica atingiu $77,6 \%$ dos participantes e verificou-se correlação negativa entre a proteína C-reativa/albumina e os demais métodos de avaliação nutricional. Conclusão: constatou-se correlação negativa fraca entre o estado nutricional e a atividade inflamatória. O diagnóstico nutricional variou entre os parâmetros de avaliação utilizados, sendo mais evidente a perda de gordura corporal.

Palavras-chave: Neoplasias pulmonares. Antropometria. Estado nutricional. Proteína C-reativa. Albuminas.

\section{ABSTRACT}

Objective: To verify the correlation between the inflammatory activity and nutritional status of patients with lung cancer. Method: Cross-sectional and quantitative study, carried out from March to October 2015 with patients with lung cancer. Patients were evaluated by body mass index, arm circumference, triceps skinfold, arm muscle circumference, total lymphocyte count and by the C-reactive protein/albumin ratio. The data were analyzed by using Spearman's correlation coefficient with significance level of 5\%. Results: A total of 58 individuals with a mean age of $65.02 \pm 9.88$ years participated. Malnutrition was higher by triceps skinfold evaluation (55.1\%) and lower by body mass index (37.9\%). Correspondingly, verified that the immunological depletion was present in $77.6 \%$ of the participants and that there was a negative correlation between the C-reactive protein/albumin ratio and the other methods of nutritional evaluation. Conclusion: There was a negative correlation between nutritional status and inflammatory activity. The nutritional diagnosis varied among the evaluation parameters used, being more evident the loss of body fat.

Keywords: Lung neoplasms. Anthropometry. Nutritional status. C-Reactive protein. Albumins.

Autor correspondente: Benedita Jales Souza, Rua Maria Ferreira dos Santos, 71, São Bento, Fortaleza, Ceará. CEP: 60875-565. Telefone: +55 85 98621-4998. E-mail: bjales.nutri@hotmail.com

Conflito de interesses: Não há qualquer conflito de interesses por parte de qualquer um dos autores.

Recebido em: 02 Abr 2018; Revisado em: 23 Mai 2018; Aceito em: 06 Jul 2018. 


\section{INTRODUÇÃO}

Em todo o mundo, a incidência de câncer vem aumentando ao longo dos anos e o tabaco é considerado o principal responsável pelo câncer de pulmão. A última estimativa do Instituto Nacional do Câncer (INCA), para o biênio 2018-2019 no Brasil, é de 31.270 casos novos de câncer de pulmão, onde espera-se atingir 18.740 homens e 12.530 mulheres. ${ }^{1}$

Aproximadamente 60 a $80 \%$ dos pacientes com este tipo de câncer apresentam caquexia no momento do diagnóstico, estando esta condição diretamente relacionada ao prognóstico adverso e redução na sobrevida. ${ }^{2}$

Por sua vez, a inflamação sistêmica desempenha papel importante na progressão da caquexia, em decorrência do aumento de hormônios contra-reguladores, citocinas pró-inflamatórias, proteína C-reativa (PCR) e redução nos níveis de albumina sérica durante o processo de carcinogênese. ${ }^{3}$

A PCR elevada é a medida mais empregada na interpretação da resposta inflamatória sistêmica em pacientes com câncer. Como as alterações inflamatórias não podem ser percebidas por meio da antropometria, tem sido importante a utilização de parâmetros que mensuram a resposta inflamatória com o intuito de identificar indivíduos com caquexia ou em risco de desenvolvê-la. ${ }^{4}$

O Índice Prognóstico Inflamatório Nutricional (IPIN) foi criado para medir o risco de complicações e/ou mortalidade relacionado a inflamação e necessita da dosagem de quatro proteínas (PCR, albumina, $\alpha-1$ glicoproteína ácida e transtiretina). ${ }^{5}$ No entanto, o elevado custo destas duas últimas proteínas tem limitado o seu uso. Diante disso, verifica-se que a fórmula PCR/Albumina utiliza marcadores de baixo custo e podem ser usados como indicadores de estado inflamatório com o mesmo poder de sensibilidade. ${ }^{6}$

Este trabalho se destaca por ser pioneiro em avaliar a atividade inflamatória pela relação PCR/Albumina em pacientes com câncer de pulmão. Com isso, objetiva-se verificar a correlação entre a atividade inflamatória e o estado nutricional de pacientes com câncer de pulmão.

\section{MATERIAL E MÉTODOS}

Trata-se de um estudo transversal e de caráter quantitativo desenvolvido com pacientes com câncer de pulmão, adultos e idosos, ambos os sexos, internados em um hospital de referência em pneumologia em Fortaleza-Ceará, entre março a outubro de 2015. Não fizeram parte do estudo indivíduos com metástase pulmonar, doença hepática crônica, pancreatite, alterações neurológicas, gestantes, amputados ou em condições que impedissem a realização da avaliação nutricional.

A participação foi confirmada com a assinatura do termo de consentimento livre e esclarecido e após aprovação do Comitê de Ética em Pesquisa com Seres Humanos do referido hospital, sob o CAAE $n^{\circ} 38984214.9 .0000 .5039$, conforme a resolução $\mathrm{n}^{\circ} 466$ de dezembro de 2012 e com base na declaração atualizada de Helsinki. ${ }^{7}$

O diagnóstico clínico e os resultados dos exames bioquímicos foram confirmados em prontuários e para a avaliação antropométrica utilizou-se o peso, altura, índice de massa corporal (IMC), circunferência do braço (CB), dobra cutânea tricipital (DCT) e circunferência muscular do braço (CMB).

O peso foi medido em balança plataforma digital (Filizola $\left.{ }^{\circledR}\right)$ e a altura aferida em estadiômetro fixado à balança. ${ }^{8} \mathrm{Na}$ presença de ascite ou edema, foi calculado o peso $\operatorname{seco}^{9}$ e para os indivíduos acamados, utilizou-se fórmulas de estimativas de peso e altura. ${ }^{10,11}$ Em seguida, o IMC foi calculado pela fórmula [IMC $\left(\mathrm{kg} / \mathrm{m}^{2}\right)=$ Peso/(Altura) $)^{2}$, respeitado os parâmetros de normalidade para adulto $\left(18,5\right.$ a $\left.24,9 \mathrm{~kg} / \mathrm{m}^{2}\right)$ e idoso $\left(22,0\right.$ a $\left.27,0 \mathrm{~kg} / \mathrm{m}^{2}\right) .{ }^{12,13}$

A CB $(\mathrm{cm})$ foi realizada no ponto médio entre o acrômio e o olécrano da ulna com o auxílio de fita métrica inelástica. Já a gordura corporal foi mensurada pela DCT (mm) com auxílio de adipômetro científico (Sanny®). Para ambos os parâmetros, foi calculada a adequação em relação ao percentil 50 , sendo considerado desnutrição $(<90 \%)$, eutrofia (90 a $110 \%)$ e excesso de peso $(>110 \%) .{ }^{14,15}$

Quanto à avaliação de reserva muscular, foi feita pela CMB pela fórmula $[\mathrm{CMB}=\mathrm{CB}$ - $(\mathrm{DCT} \times \mathrm{x}, 314)]$, sendo adotados os padrões de referência de desnutrição $(<90 \%)$ e eutrofia $(90$ a $110 \%) .^{15}$

Para a realização dos exames bioquímicos (proteína C-reativa (PCR), albumina, leucócitos e linfócitos) foram coletados $20 \mathrm{~mL}$ de sangue em veia periférica. A atividade inflamatória foi determinada pela relação PCR/albumina, sendo o estado inflamatório classificado como: normal ou sem risco de complicações $(<0,4)$, baixo risco $(0,4-1,2)$, médio risco $(1,2$ $2,0)$ e alto risco $(>2,0){ }^{6}$

A contagem total de linfócitos (CTL) $\left(\mathrm{mm}^{3}\right)$ foi utilizada para analisar a competência imunológica $[\mathrm{CTL}=(\%$ linfócitos $\mathrm{x}$ leucócitos)/100] e os resultados classificados como depleção $\left(<2.000 \mathrm{~mm}^{3}\right)$ e ausência de depleção $\left(\geq 2.000 \mathrm{~mm}^{3}\right) .{ }^{16}$

Para a análise estatística, foi empregado o software SPSS (Statistical Package for the Social Sciences, versão 22.0). As variáveis categóricas foram expressas por frequência absoluta e relativa e as numéricas por medidas de tendência central (média e desvio padrão $( \pm)$ ). Para a análise de correlação entre as variáveis utilizou-se o coeficiente de correlação de Spearman, sendo adotado o nível de significância de 5\%.

\section{RESULTADOS}

Fizeram parte do estudo 58 pacientes com idade média de $65,02 \pm 9,88$ anos, variando entre 31 a 81 anos, onde 56,9\% 
dos participantes eram do gênero feminino. Prevaleceu o baixo nível de escolaridade, com 56,9\% indivíduos possuindo o ensino fundamental incompleto e a renda familiar concentrou-se entre 1 e 2 salários mínimos na maioria dos entrevistados (65,5\%). Em relação ao estilo de vida, 67,2\% informaram ter sido fumantes e $22,4 \%$ relataram ainda fazer uso do tabaco (Tabela 1).

O IMC médio encontrado foi de $23,2 \pm 5,2 \mathrm{~kg} / \mathrm{m}^{2}$ e revelou eutrofia, embora $37,9 \%$ dos pacientes encontravam-se com baixo peso/desnutrido, $34,5 \%$ eutróficos e somente $27,6 \%$ com excesso de peso. A desnutrição foi evidenciada por todos os métodos antropométricos utilizados, sendo mais prevalente pela aferição da DCT $(55,2 \%)$ e com valores menores revelados pela CB (48,3\%), CMB (48,3\%) e IMC (37,9\%), respectivamente.

Os valores médios de PCR e albumina apresentaram-se dentro dos padrões de referência, porém, foram observadas taxas elevadas de PCR em 20,7\% dos pacientes e reduzidas de albumina em quase metade deles $(41,4 \%)$. Quanto à relação PCR/Albumina, verificou-se predomínio de atividade inflamatória normal ou sem risco de complicações entre os participantes $(34,5 \%)$, seguida de alto $(29,3 \%)$, baixo $(29,3 \%)$ e médio risco $(6,9 \%)$.

Ao se correlacionar a atividade inflamatória e o risco de complicações com as variáveis antropométricas, verificou-se correlação negativa e sem diferença significativa $(p>0,05)$, conforme ilustrado no Gráfico 1.

Verificou-se ainda que a depleção imunológica, pela CTL, foi presente em $77,6 \%$ dos indivíduos, com predomínio de depleção leve (34,5\%). A CTL também mostrou correlação negativa com a PCR/Albumina (Tabela 2) e os resultados obtidos não revelaram diferença estatística significante $(p=0,620)$.

Tabela 1. Características sociodemográficas de pacientes com câncer de pulmão internados no Hospital Dr. Carlos Alberto Studart Gomes. Fortaleza-Ceará, 2016.

\begin{tabular}{|c|c|c|}
\hline Variáveis & $\mathbf{N}$ & $\%$ \\
\hline \multicolumn{3}{|l|}{ Sexo } \\
\hline Feminino & 33 & 56,9 \\
\hline Masculino & 25 & 43,1 \\
\hline \multicolumn{3}{|l|}{ Escolaridade } \\
\hline Analfabeto & 13 & 22,4 \\
\hline Fundamental incompleto & 33 & 56,9 \\
\hline Fundamental completo & 3 & 5,2 \\
\hline Médio incompleto & 0 & 0 \\
\hline Médio completo & 7 & 12,1 \\
\hline Superior incompleto & 1 & 1,7 \\
\hline Superior completo & 1 & 1,7 \\
\hline \multicolumn{3}{|l|}{ Renda familiar* } \\
\hline$<1$ salário & 6 & 10,3 \\
\hline 1 a 2 salários mínimos & 38 & 65,6 \\
\hline$>2$ salários & 14 & 24,1 \\
\hline \multicolumn{3}{|l|}{ Tabagismo } \\
\hline Sim & 13 & 22,5 \\
\hline Não & 6 & 10,3 \\
\hline Ex-tabagista & 39 & 67,2 \\
\hline
\end{tabular}

*Valor do salário mínimo (R\$ 880,00) no período de realização do estudo. 
Gráfico 1. Correlação de PCR/Albumina com variáveis antropométricas de pacientes internados com câncer de pulmão. FortalezaCeará, 2015.

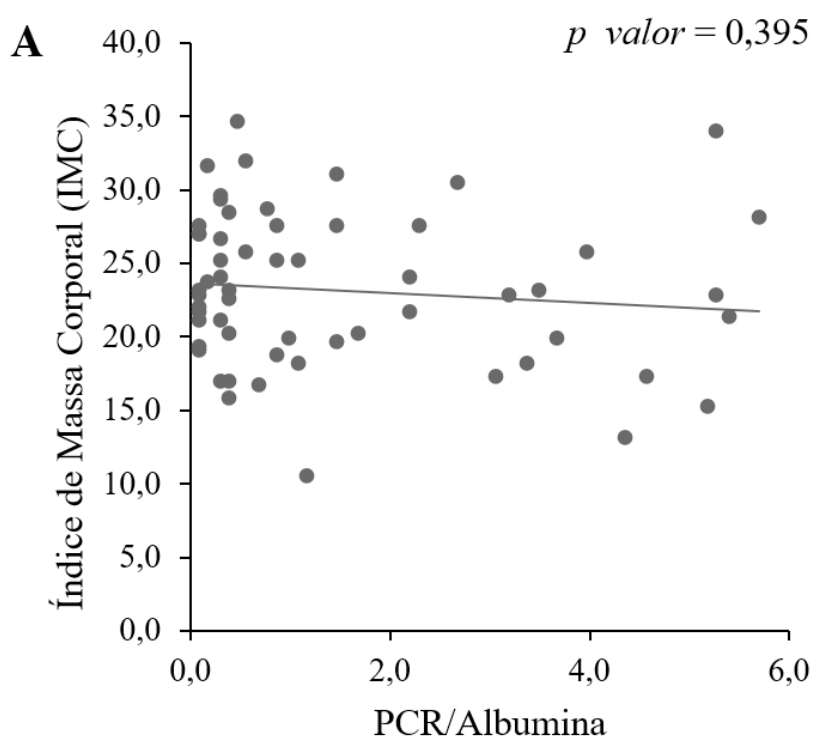

- $\operatorname{IMC}\left(\mathrm{kg} / \mathrm{m}^{2}\right) \quad$ Linear $\left(\operatorname{IMC}\left(\mathrm{kg} / \mathrm{m}^{2}\right)\right)$

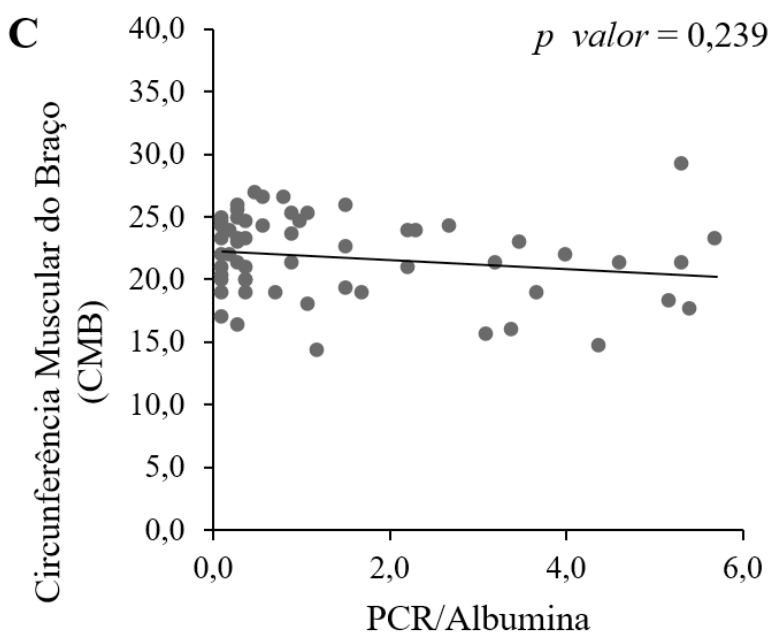

- $\mathrm{CMB}(\mathrm{cm}) \quad$ Linear $(\mathrm{CMB}(\mathrm{cm}))$

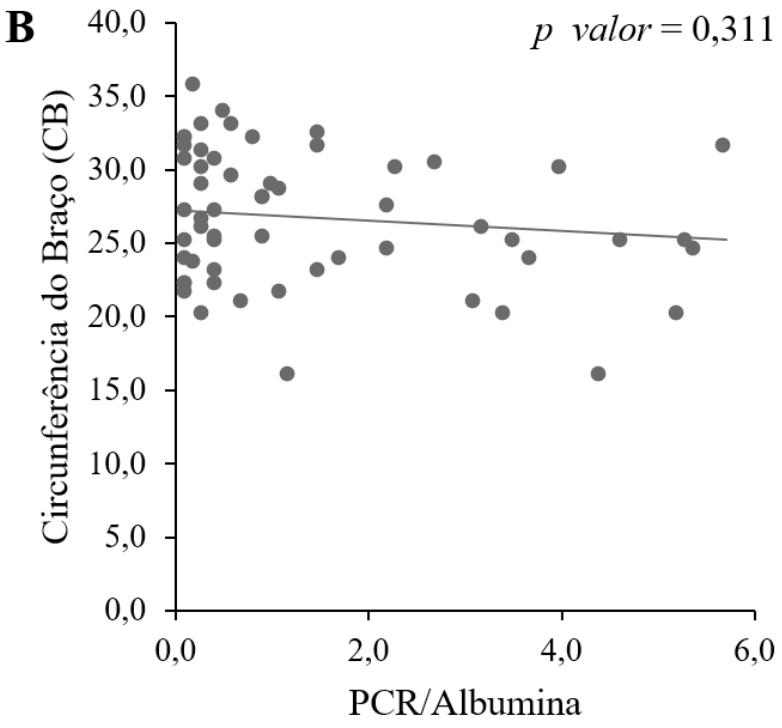

- $\mathrm{CB}(\mathrm{cm}) \quad$ Linear $(\mathrm{CB}(\mathrm{cm}))$

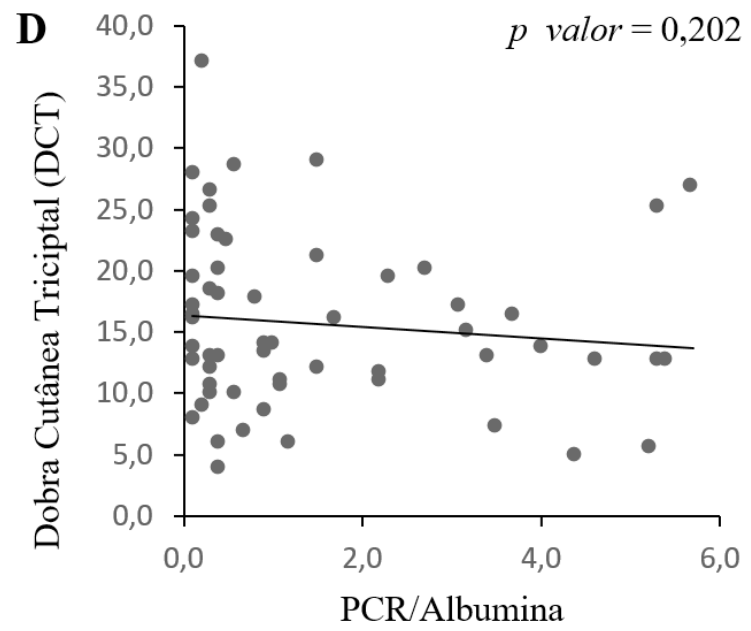

- $\operatorname{DCT}(\mathrm{mm}) \quad$ Linear (DCT $(\mathrm{mm}))$

Tabela 2. Correlação entre PCR/Albumina e diferentes métodos de avaliação nutricional de pacientes com câncer de pulmão. FortalezaCeará, 2015.

\begin{tabular}{|c|c|c|c|c|c|}
\hline \multirow{2}{*}{ Variáveis } & IMC (kg/m²) & $\mathrm{CB}(\mathrm{cm})$ & CMB (cm) & DCT (mm) & $\operatorname{CTL}\left(\mathrm{mm}^{3}\right)$ \\
\hline & $\rho$ & $\rho$ & $\rho$ & $\mathrm{P}$ & $\mathrm{P}$ \\
\hline PCR/Albumina & $-0,114$ & $-0,135$ & $-0,157$ & $-0,170$ & $-0,247$ \\
\hline
\end{tabular}




\section{DISCUSSÃO}

O tabaco continua sendo a principal causa de câncer de pulmão em todo o mundo e, como visto neste trabalho, o percentual de fumantes e ex-fumantes foi bastante elevado (89,7\%), superior até ao encontrado por Gioulbasanis et al (74\%). ${ }^{17}$

Gioulbasanis et $\mathrm{al}^{17} \mathrm{e} \mathrm{Kim}$ et $\mathrm{al},{ }^{18}$ investigando mais de 100 pacientes, encontraram predomínio de câncer de pulmão em homens, respectivamente, $87,8 \%$ e $83,9 \%$. Contrariando tais estudos, no presente trabalho foi mais evidente no sexo feminino $(56,9 \%)$, tal fato pode ser explicado pelo aumento no número de mulheres fumantes nas últimas décadas.

Com relação à avaliação nutricional, sabe-se que ela é necessária para identificar os pacientes em risco de desenvolver desnutrição, assim como servir de base para o planejamento e o tratamento dietoterápico adequado. ${ }^{19}$

O índice de massa corporal é um dos métodos de avaliação mais utilizado na prática clínica e em estudos epidemiológicos, sendo inclusive preconizado para pacientes oncológicos por ser de fácil aplicação e de baixo custo. ${ }^{20}$ Apesar destes pacientes serem mais vulneráveis à perda de peso, tal vulnerabilidade não pôde ser evidenciada pelo IMC neste estudo, uma vez que o IMC médio revelou eutrofia, estando dentro da faixa de normalidade com $23,2 \pm 5,2 \mathrm{~kg} / \mathrm{m}^{2}$, corroborando com outras pesquisas, respectivamente, $22,44 \pm 4,06 \mathrm{~kg} / \mathrm{m}^{2}$ e $24,8 \pm 4,5$ $\mathrm{kg} / \mathrm{m}^{2} .^{21,22}$ Tal resultado pode refletir a limitação do IMC em não ser capaz de identificar alterações nos tecidos adiposo e muscular, bem como no estado de hidratação. ${ }^{20}$

Desnutrição é uma complicação frequentemente observada em pacientes com câncer, em virtude do aumento das necessidades energéticas, ingestão inadequada de alimentos e/ou dificuldade na absorção de nutrientes. ${ }^{20}$ Essa ocorrência pôde ser confirmada pela aferição da circunferência do braço, que revelou desnutrição em quase metade dos participantes $(48,3 \%)$ deste estudo, embora o valor médio de $26,7 \pm 4,7 \mathrm{~cm}$ estivesse dentro do intervalo encontrado em um estudo prospectivo envolvendo 30 pacientes. ${ }^{21}$

A circunferência muscular do braço permite estimar a reserva proteica, também sendo considerada como indicador precoce de depleção nutricional. Estudo desenvolvido com 56 pacientes com câncer de pulmão de não pequenas células, em estágio IV, encontrou $55 \%$ de desnutrição por esse método de avaliação, ${ }^{23}$ percentual superior a 48,3\% obtido neste trabalho. Embora a perda de massa muscular tenha sido um achado comum nesses indivíduos, foi a DCT o parâmetro que mais diagnosticou desnutrição $(55,1 \%)$.

A avaliação imunológica, a partir da contagem total de linfócitos, mostrou depleção na maioria dos participantes $(77,6 \%)$. Fato este que pode ser atribuído às alterações inflamatórias presentes no câncer e que provocam mudanças nas defesas imunológicas. ${ }^{24}$

Diferentemente da desnutrição, a caquexia é uma síndrome gerada por alterações hormonais com consequência no metabolismo dos nutrientes e, principalmente, pela ativação de citocinas inflamatórias que são responsáveis por intenso catabolismo proteico, podendo estar associada à perda de tecido adiposo, anemia e astenia. ${ }^{25}$ Porém, muitos estudos têm se limitado ao uso da antropometria como forma de avaliação nutricional e tem se visto que a mensuração de marcadores de estado inflamatório não tem ganhado a mesma importância. ${ }^{19}$

A proteína C-reativa é um marcador importante na investigação da inflamação em pacientes com câncer. Kim et al em seu estudo com 186 pacientes encontrou ligação entre PCR >10g/dL e perda de massa muscular. ${ }^{18}$ Semelhantemente no presente trabalho, foi observado aumento de PCR em 20,7\% dos pacientes e a perda de peso em quase metade dos participantes da pesquisa.

A albumina é a proteína mais abundante no plasma e importante para o transporte de medicamentos. ${ }^{26}$ Neste estudo, $41,4 \%$ dos pacientes apresentaram hipoalbuminemia, embora o valor médio de albumina $(3,5 \pm 0,5 \mathrm{~g} / \mathrm{dL})$ tenha se mantido no limite mínimo recomendado. Contradizendo tais resultados, um estudo multicêntrico com 86 pacientes, identificou valores inferiores de albumina sérica $(3,1 \mathrm{~g} / \mathrm{dL})$ entre os participantes. ${ }^{24}$

Uma revisão sistemática revelou que de dez trabalhos avaliados, nove mostraram albumina superior a 3,5g/dL antes do tratamento antineoplásico e que esta condição está associada a melhor prognóstico. ${ }^{27}$ Pensando nisso, acredita-se que o combate à hipoalbuminemia poderia ter efeito satisfatório na sobrevida.

Em pesquisa publicada com 303 pacientes, observou-se que mesmo após a ressecção do tumor, os níveis de PCR e albumina, quando avaliados separadamente, apresentaram correlação oposta. ${ }^{28}$

Devido à ausência de estudos utilizando a relação PCR/ Albumina em pacientes com câncer de pulmão, nesta pesquisa buscou-se correlacionar todas as variáveis utilizadas com a atividade inflamatória proposta pela fórmula PCR/Albumina e constatou-se também correlação inversa, isto é, os pacientes que apresentaram menores valores de IMC, CB, DCT, CMB e CTL, foram os que tiveram maior resposta inflamatória e, consequentemente, riscos de complicações.

\section{CONCLUSÃO}

$\mathrm{Na}$ amostra estudada, evidenciou-se correlação negativa entre a atividade inflamatória e o estado nutricional, mostrando que a inflamação foi mais evidente nos pacientes com menores valores antropométricos.

O diagnóstico nutricional variou entre os parâmetros de avaliação utilizados, sendo mais evidente a desnutrição com perda de gordura corporal.

Diante das evidências científicas sobre o impacto da inflamação no estado nutricional de pacientes oncológicos, reforça-se a necessidade de mensuração de marcadores bioquímicos como parte da avaliação nutricional desses indivíduos e de mais pesquisas para confirmar os dados achados neste estudo. 


\section{REFERÊNCIAS}

1. Instituto Nacional do Câncer José Alencar Gomes da Silva (INCA). Estimativa 2018: incidência de câncer no Brasil [Internet]. Rio de Janeiro (RJ): INCA; 2017 [acesso em: 16 Jan 2018]. Disponível em: http://www1.inca.gov.br/inca/Arquivos/estimativa-2018.pdf

2. Associação Brasileira de Cuidados Paliativos (ABCP). Consenso brasileiro de caquexia/anorexia em cuidados paliativos. Revista Brasileira de Cuidados Paliativos. 2011;3(3 Supl 1):4-23.

3. Pastore CA, Orlandi SP, Gonzalez MC. The inflammatory-nutritional index; assessing nutritional status and prognosis in gastrointestinal and lung cancer patients. Nutr Hosp. 2014;29(3):629-34.

4. McMillan DC. Systemic inflammation, nutritional status and survival in patients with câncer. Curr Opin Clin Nutr Metab Care. 2009:12(3):223-6.

5. Ingenbleek Y, Carpentier YA. A prognostic inflammatory and nutritional index scoring critically ill patients. Int J Vitam Nutr Res. 1985;55(1):91-101.

6. Corrêa CR, Angeleli AY, Camargo NR, Barbosa L, Burini RC. Comparação entre a relação PCR/albumina e o índice prognóstico inflamatório nutricional (IPIN). J Bras Patol Med Lab. 2002:38(3):183-90.

7. World Medical Association Declaration of Helsinki. Ethical principles for medical research involving human subjects. Bull World Health Organ. 2001;79(4):373-4.

8. Brasil. Ministério da Saúde [Internet]. Orientações para a coleta e análise de dados antropométricos em serviços de saúde. Brasília (DF); 2011. [Acesso em 7 mar 2017]. Disponível em: http://bvsms. saude.gov.br/bvs/publicacoes/orientacoes_coleta_analise_dados antropometricos.pdf

9. Martins C, Cardoso SP. Terapia nutricional enteral e parenteral: manual de rotina técnica. Curitiba: Nutroclínica; 2000.

10. Chumlea WC, Roche AF, Steinbaugh ML. Estimating stature from knee height for persons 60 to 90 years of age. J Am Geriatr Soc. 1985;33(2):116-20.

11. Chumlea WC, Guo S, Roche AF, Steinbaugh ML. Prediction of body weight for the nonambulatory elderly from anthropometry. J Am Diet Assoc. 1988;88(5):564-8.

12. World Health Organization (WHO). Physical Status: the use and interpretation of anthropometry. Gèneve: World Heath Organization; 1995.

13. Lipschitz DA. Screening for nutritional status in the elderly. Prim Care. 1994;21(1):55-67.

14. Frisancho AR. Antropometric Standards for the assessment of growth and nutritional status. Michigan: The University of Michigan Press; 1990.

15. Blackburn GL, Thornton PA. Nutritional assessment of the hospitalized patient. Med Clin North Am. 1979;63(5):11103-15.
16. Blackburn GL, Bistrian BR, Maini BS, Schlamn HT, Smith MF. Nutritional and metabolic assessment of the hospitalized patient. J. Parenter Enteral Nutr. 1977;1(1):11-22.

17. Gioulbasanis I, Georgoulias P, Vlachostergios PJ, Baracos V, Ghosh S, Giannousi Z, et al. Mini Nutritional Assessment (MNA) and biochemical markers of cachexia in metastatic lung cancer patients: Interrelations and associations with prognosis. Lung Cancer. 2011;74(3):516-20.

18. Kim EY, Kim YS, Seo JY, Park I, Ahn HK, Jeong YM, et al. The relationship between sarcopenia and systemic inflammatory response for cancer cachexia in small cell lung cancer. Plos One. 2016;11(8):110

19. Huhmann MB, Cunningham RS. Importance of nutritional screening in treatment of cancer-related weight loss. Lancet Oncol. 2005;6(5):334-43.

20. Instituto Nacional de Câncer José Alencar Gomes da Silva (INCA) [Internet]. Consenso nacional de nutrição oncológica. Rio de Janeiro (RJ): INCA; 2016. [acesso em: 10 jan 2017]. Disponível em: http://wwwl.inca.gov.br/inca/Arquivos/Consenso_Nutricao_vol_ II_2_ed_2016.pdf

21. Chermiti BA, Ben SH, Chamkhi N, Ferchichi M, Chtourou A, Taktak S, et al. Assessment of nutritional status in patients with primary lung cancer. Tunis Med. 2013;91(10):600-4.

22. Sánchez-Lara K, Turcott JG, Juárez E, Guevara P, Núñez-Valencia $C$, Oñate-Ocaña LF, et al. Association of nutrition parameters including bioelectrical impedance and systemic inflammatory response with quality of life and prognosis in patients with advanced non-small-cell lung cancer: a prospective study. Nutr Cancer. 2012;64(4):526-34.

23. Tartari R, Busnello F, Nunes CH. Perfil nutricional de pacientes em tratamento quimioterápico em um ambulatório especializado em quimioterapia. Rev Bras Cancerol. 2010;56(1):43-50.

24. Bagan P, Berna P, Dominicis F, Pereira JC, Mordant P, La Tour B, et al. Nutritional status and postoperative outcome after pneumonectomy for lung cancer. Ann Thorac Surg. 2013;95(2):392-6.

25. Laviano A, Koverech A, Mari A. Cachexia: clinical features when inflammation drives malnutrition. Proc Nutr Soc. 2015;74(4):348-54.

26. Wang X, Han H, Duan Q, Khan U, Hu Y, Yao X. Changes of serum albumin level and systemic inflammatory response in inoperable non-small cell lung cancer patients after chemotherapy. J Cancer Res Ther. 2014;10(4):1019-23.

27. Gupta D, Lis CG. Pretreatment serum albumin as a predictor of cancer survival: a systematic review of the epidemiological literature. Nutr J. 2010;69(9):1-16.

28. Alifano M, Mansuet-Lupo A, Lococo F, Roche N, Bobbio A, Canny E, et al. Systemic inflammation, nutritional status and tumor immune microenvironment determine outcome of resected non-small cell lung cancer. Plos One. 2014;9(9):1-11.

\section{Como citar:}

Souza BJ, Mesquita AU, Meireles AR, Brito JG, Bandeira TE, Rocha JA. Relação entre a atividade inflamatória e o estado nutricional de pacientes com câncer de pulmão. Rev Med UFC. 2019 abr-jun;59(2):9-14. 